Meta

Journal des traducteurs

Translators' Journal

\title{
Mise en ligne d'outils pédagogiques : une panacée pour l'enseignement de la traduction médicale ?
}

\section{Sylvie Vandaele}

Volume 48, numéro 3, septembre 2003

Traduction et enseignement

Translation and teaching

URI : https://id.erudit.org/iderudit/007597ar

DOI : https://doi.org/10.7202/007597ar

Aller au sommaire du numéro

Éditeur(s)

Les Presses de l'Université de Montréal

ISSN

0026-0452 (imprimé)

1492-1421 (numérique)

Découvrir la revue

Citer cet article

Vandaele, S. (2003). Mise en ligne d'outils pédagogiques : une panacée pour l'enseignement de la traduction médicale ? Meta, 48(3), 370-378.

https://doi.org/10.7202/007597ar
Résumé de l'article

Les contraintes de l'enseignement de la langue et de la traduction médicales résultent non seulement de la complexité de la matière, mais aussi de la conjugaison de deux facteurs antagonistes : d'une part, les acquis préalables des étudiants sont hétérogènes ; d'autre part, les exigences du marché du travail sont élevées. Le présent article fait état de la mise en oeuvre de sites utilisant la plateforme WebCT, qui permet la mise en ligne de différents outils pédagogiques. L’objectif est de compléter les cours donnés en classe et d'ajouter à l'approche pédagogique traditionnelle des outils qui favorisent la progression individuelle de l'étudiant et lui offrent une mise en situation dans des conditions évoquant la vie professionnelle. 


\title{
Mise en ligne d'outils pédagogiques: une panacée pour l'enseignement de la traduction médicale?
}

\author{
SYLVIE VANDAELE \\ Université de Montréal, Montréal, Canada \\ sylvie.vandaele@umontreal.ca
}

\begin{abstract}
RÉSUMÉ
Les contraintes de l'enseignement de la langue et de la traduction médicales résultent non seulement de la complexité de la matière, mais aussi de la conjugaison de deux facteurs antagonistes: d'une part, les acquis préalables des étudiants sont hétérogènes; d'autre part, les exigences du marché du travail sont élevées. Le présent article fait état de la mise en œuvre de sites utilisant la plateforme WebCT, qui permet la mise en ligne de différents outils pédagogiques. L'objectif est de compléter les cours donnés en classe et d'ajouter à l'approche pédagogique traditionnelle des outils qui favorisent la progression individuelle de l'étudiant et lui offrent une mise en situation dans des conditions évoquant la vie professionnelle.
\end{abstract}

\section{ABSTRACT}

The obstacles encountered in teaching both medical language and medical translation are two-pronged. On the one hand, the students' backgournd knowledge in both translation and the healthcare field is very varied. On the other hand, professional medical translation is very demanding. This article focuses on the development of didactic material using WebCT. This computerized material is meant to enhance teaching and learning, but not to replace traditional classroom work, and also to stimulate the progress of each student individually in real-life professional situations.

\section{MOTS-CLÉS/KEYWORDS}

langue médicale, traduction médicale, NTIC, WebCT, outils pédagogiques

Depuis quelques années, les universités font l'acquisition de plateformes informatiques permettant la mise en ligne de différents outils pédagogiques ${ }^{1}$. Les universités québécoises ne sont pas en reste, puiqu'elles ont mis en œuvre des politiques et des infrastructures visant à intégrer les «nouvelles technologies de l'information et des communications» (NTIC). À l'instar de plusieurs autres universités ${ }^{2}$, l'Université de Montréal a choisi, parmi les différents logiciels proposés, WebCT ${ }^{3}$, dont le développement a été initialement assuré par la University of British Columbia (Giroux 1999: 35). L'intégration de cette plateforme informatique dans les pratiques des professeurs est soutenue par un programme spécial ${ }^{4}$.

La variété des outils offerts par WebCT permet de multiples applications pédagogiques, que ce soit dans le cadre de cours à distance ou à titre de complément au cours donné en classe. La Florida International University, pour sa part, offre un cours de traduction médicale à distance, espagnol-anglais (Gómez et Weinberg $2002)^{5}$. En ce qui nous concerne, nous utilisons WebCT pour compléter les différents 
cours de langue et de traduction médicales (anglais-français) offerts dans nos programmes. Dans le présent article, nous visons à expliciter comment certains outils de WebCT permettent de répondre à des objectifs pédagogiques spécifiques à l'enseignement de la langue et de la traduction médicales.

\section{Contexte pédagogique}

L'Université de Montréal offre plusieurs cours de langue et de traduction médicales destinés au premier et au deuxième cycles ${ }^{6}$. Le cours portant sur la langue médicale compte de cinquante à soixante étudiants et rassemble les groupes qui suivront ensuite le cours de traduction médicale de premier ou de deuxième cycles (DESS), cours qui comptent chacun une vingtaine d'étudiants. Le cours de traduction médicale offert à la maîtrise est suivi par des étudiants venant du baccalauréat ou du DESS, ou encore ayant suivi un programme de traduction dans une autre université; il regroupe entre dix et vingt étudiants. En tout, comme certains suivent plusieurs cours, de soixante à quatre-vingt dix étudiants sont concernés chaque année.

La caractéristique essentielle de ces cohortes est l'hétérogénéité: en effet, tous n'ont pas la même formation préalable. La variété des cursus antérieurs est plus particulièrement perceptible au deuxième cycle. Au premier cycle, c'est sutout l'hétérogénéité de l'intégration des connaissances traductionnelles et terminologiques qui se fait sentir. Dans tous les cas, rares sont ceux qui ont déjà suivi des cours en science ou dans les disciplines médicales ou paramédicales. Enfin, certains mythes doivent être combattus, tels que la croyance qu'il n'est pas nécessaire d'étudier dans un cours de traduction, qu'être bilingue équivaut à être automatiquement compétent en traduction, ou que Google et Internet résoudront tous les problèmes, notionnels ou terminologiques. À l'inverse, mais de moins en moins fréquemment, certains ne se sentent pas concernés par l'informatique, ou la redoutent.

Par ailleurs, la matière elle-même - médecine et sciences connexes - est vaste et elle a déjà fait l'objet de plusieurs réflexions en matière de pédagogie de la traduction (Balliu 1994; Rouleau 1994; Lee-Jahnke 2001; Vandaele, 2001). Rappelons simplement de nombreux sous-domaines complexes y participent, que les connaissances évoluent rapidement et que, sans vouloir prétendre à une quelconque supériorité sur les autres spécialités, la santé humaine est bien souvent en jeu dans les textes abordés: la responsabilité du traducteur est donc lourde.

Le défi est par conséquent de taille: préparer l'étudiant au marché de la traduction médicale, ce n'est pas lui donner un cours de médecine condensé en une ou plusieurs sessions. Il s'agit plutôt de l'amener à une autonomie intellectuelle lui permettant de prendre avec aisance ses propes décisions traductionnelles dans les délais requis. Par conséquent, les objectifs pédagogiques viseront à renforcer les acquis de la pratique de la traduction et de la terminologie normalement vus dans d'autres cours, ainsi qu'à introduire les spécificités du domaine: non seulement est-il indispensable d'aborder des notions de base nécessaires à la construction des connaissances ultérieures - ce que nous avons appelé les «noyaux conceptuels» (Vandaele 2001) -, mais également les particularités des textes et de la langue, ainsi que la documentation et les outils informatiques pertinents. Ainsi, en une ou plusieurs sessions de 45 heures, il faut répondre à ces interrogations: comment favoriser la progression 
individuelle des étudiants, voire leur mise à niveau? Comment favoriser la construction d'un savoir dans un domaine souvent nouveau, sans toutefois aborder les notions de manière systématique? Comment initier les étudiants à la documentation sans les «noyer»? Et surtout, comment favoriser la construction d'un sens critique?

Soulignons tout d'abord que les moyens pédagogiques employés traditionnellement demeurent toujours pertinents: traduction de textes représentatifs du marché du travail et traitant des grands axes de recherche médicale actuels (cancer, sida, etc.), réflexion sur des particularités du domaine (terminologie, usages...), recherche documentaire. Alors, à quoi WebCT peut-il servir pour répondre aux besoins ainsi identifiés?

\section{Utilisation de WebCT}

TABLEAU 1

Objectifs visés dans le choix des outils WebCT

\begin{tabular}{|c|c|}
\hline OBJECTIFS VISÉS & OUTIL WEBCT \\
\hline \multicolumn{2}{|l|}{ A. Objectifs généraux } \\
\hline $\begin{array}{l}\text { - Structuration du cours } \\
\text { - Contenu du cours } \\
\text { - Correction des traductions } \\
\text { - Communication entre étudiants } \\
\text { et avec la professeure }\end{array}$ & $\begin{array}{l}\text { - Affichage du plan de cours } \\
\text { - Calendrier } \\
\text { - Section dédiée à l'affichage et à la remise } \\
\text { des travaux } \\
\text { - Notes de cours (résumés) } \\
\text { - Courriel }\end{array}$ \\
\hline \multicolumn{2}{|l|}{ B. Objectifs spécifiques } \\
\hline $\begin{array}{l}\text { Maîtrise de l'accès aux ressources } \\
\text { documentaires du domaine }\end{array}$ & $\begin{array}{l}\text { - Liens vers les bibliothèques pertinentes, } \\
\text { les banques de données en ligne (Medline, } \\
\text { PubMed, le Grand dictionnaire } \\
\text { terminologique) }\end{array}$ \\
\hline $\begin{array}{l}\text { - Connaissance des ressources documentaires } \\
\text { du domaine }\end{array}$ & $\begin{array}{l}\text { Liens HTML ou affichage de textes } \\
\text { pertinents: bulletins terminologiques } \\
\text { ou textes traitant des notions } \\
\text { - Mode d'emploi des banques de données } \\
\text { telles que Medline }\end{array}$ \\
\hline $\begin{array}{l}\text { - Familiarisation des étudiants avec un usage } \\
\text { raisonné des fureteurs et d'Internet } \\
\text { - Orientation sur des liens pertinents }\end{array}$ & - Fichiers d'aides \\
\hline - Gestion des fichiers informatiques & \\
\hline & $\begin{array}{l}\text { Boîte de réception des travaux (dates } \\
\text { butoirs) }\end{array}$ \\
\hline - Acquisition de la terminologie médicale & $\begin{array}{l}\text { - Exercices à préparer pour le cours suivant } \\
\text { affichés sur le site (ou référence au cahier } \\
\text { d'exercices) } \\
\text { - Quiz en ligne non notés (entraînement } \\
\text { et préparation aux examens) ou notés } \\
\text { (avec correction automatique) }\end{array}$ \\
\hline $\begin{array}{l}\text { - Maîtrise du métalangage de la traduction } \\
\text { et de la terminologie }\end{array}$ & $\begin{array}{l}\text { - Glossaire des principaux termes employés } \\
\text { dans le cours }\end{array}$ \\
\hline
\end{tabular}


Il faut savoir, tout d'abord, que WebCT se présente, pour le concepteur, comme une «boîte à outils» dans laquelle il puisera les fonctionnalités dont il a besoin en fonction de sa stratégie pédagogique ${ }^{7}$. Ainsi, à partir de la page d'accueil du site dédié au cours, l'étudiant a directement accès à des outils lui permettant de repérer la structure du cours ou ayant trait au processus de traduction, ainsi qu'à de la documentation en ligne et à des outils de communication. Certains outils ne nous ont pas paru pertinents dans notre contexte pédagogique, comme la mise en ligne de vidéos, ou trop lourds à gérer, comme le clavardage.

Voyons un peu plus en détail les outils utilisés, et quels sont les objectifs sousjacents à leur sélection (Tableau 1). Tout d'abord, le site facilite la structuration du cours. Ainsi, le plan de cours est affiché à partir de la page d'accueil. Il reproduit bien entendu le plan de cours sur papier remis en début de session, mais se montre plus «dynamique», puisque les différentes parties sont reliées par des liens, et que l'on peut accéder à des sites externes, comme la bibliothèque. Ensuite, WebCT offre un calendrier dans lequel le professeur a la possiblité d'indiquer les dates-clés de la session (remises de travaux, examens, par exemple). Enfin, une section est consacrée spécifiquement aux travaux («boîte de réception des travaux»). Elle permet d'afficher le descriptif des travaux demandés, les dates limites, et elle offre la possibilité de télécharger des documents s'y rapportant (le texte à traduire, par exemple). Il est possible de configurer le logiciel afin que les travaux s'affichent à partir d'une certaine date et que l'accès de la boîte de réception soit interdite, pour un travail donné, à partir d'une date butoir.

La section relative aux travaux touche d'ailleurs aux trois axes énoncés plus haut: non seulement, comme nous venons de le voir, elle permet de structurer le cours, mais elle intervient dans la gestion du processus de traduction et dans la communication entre étudiant et professeur. En effet, l'étudiant retourne un fichier Word dans la boîte de dépôt des travaux, fichier ensuite récupéré par le professeur. Après correction, le fichier est retourné à l'étudiant. Par conséquent, l'ensemble des contraintes reproduit schématiquement certaines des conditions de travail futures: il impose à l'étudiant (parfois avec des grincements de dents, mais le plus souvent avec enthousiame, surtout au second cycle) de se familiariser avec le maniement d'outils informatiques de communication, de lire les instructions et, il faut bien le reconnaître, de gérer un certain stress relié à l'usage de ces outils. Il faut souligner aussi que, parmi les personnes un peu réticentes au départ, certaines nous ont témoigné, après s'être frottées au monde du travail par l'intermédiaire des stages, qu'il était fort utile de se familiariser avec les TIC au cours de la formation universitaire...

Par ailleurs, WebCT permet certes d'afficher les textes à traduire, mais aussi, si on le désire, les corrigés ou des notes relatives aux difficultés rencontrées au cours de la correction, sous la forme de pages en format HTML. Il est maintenant assez facile d'éditer de telles pages, puisque les logiciels actuels autorisent une saisie indépendante de l'affichage des codes HTML.

Les particularités du domaine en matière de terminologie et de documentation sont également illustrées à l'aide du site, en complément au cours et aux discussions en classe. L'étudiant aura ainsi facilement accès à une base documentaire qui lui permettra d'approfondir certains problèmes abordés oralement, mais aussi d'approfondir sa connaissance du domaine, en consultant, voire en téléchargeant, la documentation mise à sa disposition. Nous avons eu toutefois à faire face à un inconvénient que 
nous n'avions pas prévu au départ: lors de la sélection des documents, nous suivons les règles de Copibeq en matière de photocopies d'articles et d'extraits d'ouvrages et nous pensions que respecter ces limitations nous autoriserait à mettre en ligne lesdits extraits, comme si nous mettions un recueil en format papier à la réserve de la bibliothèque. Toutefois, en raison du vide juridique lié à l'affichage en ligne, nous nous sommes aperçue qu'il fallait demander les autorisations idoines aux éditeurs concernés, ce qui a considérablement alourdi la tâche. De fait, un certain nombre d'autorisations ont été obtenues, et les documents correspondants ont été affichés, mais nous avons été obligée de garder la formule du recueil, du moins temporairement. Par contre, la puissance de l'affichage en ligne se révèle lorsqu'il s'agit d'indiquer des liens intéressants (accès à des banques de terminologie sur Internet, à des bibliothèques, ou à des sites spécialisés de qualité). À ce chapitre, certains étudiants se sont montrés très enthousiastes et n'ont pas hésité à faire part au groupe de leurs trouvailles, tant au plan terminologique qu'en matière de ressources documentaires. Les liens qu'ils nous font parvenir, après vérification, sont rassemblés dans un fichier appelé «Les bonnes adresses de la Toile médicale», ce qui les rend facilement utilisables par tous de session en session.

Nous avons vu plus haut que la section relative aux travaux (réception, puis retour des corrections) faisait partie de l'aspect communicationnel de la stratégie pédagogique. Deux autres outils ont été installés dans chacun des sites: le courriel et le forum de discussion. Le courriel a eu un succès certain. Les étudiants étant automatiquement inscrits en début de session, ni eux ni le professeur n'ont à se préoccuper de recueillir les adresses de courriel. Les échanges professeur-étudiant s'en trouvent facilités. Loin de générer une mise à distance liée à l'usage d'outils informatiques somme toute impersonnels, ils ont souvent permis de «briser la glace» et d'instaurer avec un certain nombre d'étudiants un contact plus personnalisé. N'ayant bien entendu pas accès aux échanges entre les étudiants, nous ne pouvons juger de l'ampleur de l'usage du courriel au sein du groupe, mais c'est une question qui pourra éventuellement être posée explicitement plus tard.

Par contre, le forum, qui se voulait plus spécifiquement un espace permettant de débattre de difficultés rencontrées dans les travaux, n’a pas recueilli de suffrages. Il est certain que le manque de temps en est le principal responsable. Il faudrait peutêtre, pour qu'il soit productif, qu'il soit activement géré par un responsable, comme pour les listes de diffusion accessibles sur Internet, ou que les interventions fassent l'objet d'une note (participation au cours), mais la charge de travail nous a éloignée de cette voie quelque peu chronophage et, au demeurant, d'importance moindre sur le plan pédagogique.

Dans le cours de langue médicale, nous avons eu recours à un outil particulièrement prometteur: les quiz. Il s'agit de questionnaires en ligne, formule qui particulièrement bien adaptée à l'apprentissage d'un vocabulaire spécialisé. Différentes formes de questionnaires sont offertes: questions à choix multiples, phrases à compléter, mises en correspondances, questions à réponse courte ou longue. Le quiz peut être noté ou non. Il peut donc être utilisé, dans un premier temps, à titre d'entraînement $^{8}$. Dans un deuxième temps, il peut être utilisé comme examen, le système se chargeant de la correction des copies, ce qui est loin d'être négligeable lorsque les groupes comptent plus de cinquante étudiants. Toutefois, la correction automatique 
impose d'exclure les questionnaires faisant appel à des réponses longues, non reproductibles d'un étudiant à l'autre.

Le dernier outil que nous avons choisi d'utiliser est le glossaire. D'une part, les étudiants éprouvent une certaine difficulté à maîtriser le métalangage de la traduction et de la terminologie. Ils ont souvent besoin d'approfondir la compréhension des erreurs qu'ils ont commises (Qu'est-ce qu'au juste qu'un contresens, un faux sens ou un non-sens?). D’autre part, comme nous l'avons déjà mentionné, le niveau de leurs connaissances antérieures est hétérogène. Il est donc particulièrement utile d'avoir sous la main un ensemble des principaux termes dont ils auront besoin pour expliquer et commenter leur travail de recherche documentaire, de terminologie et de traduction. Nous espérons ainsi favoriser l'ancrage des connaissances acquises dans les cours antérieurs. Le glossaire est accessible soit directement, soit par l'intermédiaire d'hyperliens dans des pages affichées dans le site.

\section{Difficultés rencontrées}

Bien entendu, la mise en œuvre d'un nouvel outil pédagogique faisant appel aux TIC n'est pas sans imposer un certain nombre de contraintes (Lamoureux, 1999). Outre le fait que le concepteur (le professeur en l'occurrence) doit se former à l'utilisation de la plateforme ${ }^{9}$, il faut encore en faire la démonstration du fonctionnement aux étudiants en début d'année. Nous avons été la première à mettre en place un cours sur WebCT dans notre département ${ }^{10}$. L'innovation a un prix: il faut apprendre aux étudiants à entrer dans le site et à y naviguer, gérer la remise des mots de passe, bâtir un fichier d'aide guidant les étudiants dans la configuration de leur fureteur et dans l'exploration du site. Grâce à deux subventions ${ }^{11}$, nous avons pu rémunérer des auxiliaires qui, non seulement ont aidé à la réalisation des différents sites, mais ont également consacré du temps à répondre aux questions techniques des étudiants. Nous avons évalué, globalement, que $16 \%$ des étudiants ont posé des questions techniques durant la première session de fonctionnement. En fait, d'année en année, le problème lié aux difficultés techniques risque de s'atténuer, car d'autres cours font maintenant appel à WebCT et il est probable que les étudiants réfractaires à l'utilisation de l'informatique à l'université se raréfient. Nous pensons donc que les efforts de formation des étudiants à l'utilisation du site devraient être moins lourds dans l'avenir.

Parmi les autres contraintes, il faut remarquer que le recours au site impose aux étudiants d'avoir accès à un matériel informatique performant. Le Rapport Giroux mentionne qu'au printemps 1999, $87 \%$ des étudiants de notre faculté disposaient d'un ordinateur à domicile, tandis que $53 \%$ étaient abonnés à un fournisseur Internet, sans compter l'accès sur le campus (Giroux 1999: 28). L'acquisition d'un ordinateur est de plus en plus la norme, surtout en traduction, et il est probable que le pourcentage d'étudiants disposant d'un accès Internet augmente d'année en année. Par ailleurs, des efforts pour élargir le parc d'ordinateur ont été consentis par notre université. De plus, l'accès à WebCT peut se faire à distance de n'importe quel poste de travail, ce qui n'impose pas de réserver une salle d'ordinateur spécifique. En ce qui nous concerne, comme nous l'avons mentionné plus haut, nous nous sommes sentie limitée dans les possibilités d'affichage de la documentation pertinente et nous avons 
parfois trouvé que certaines fonctions de WebCT mériteraient d'être allégées (par exemple, les étapes de téléchargement de fichier). Nous espérons également que la compatibilité avec les différents fureteurs s'améliore: d'un côté, l'impression des pages HTML est parfois incomplète avec Netscape (version 4.7), de l'autre, l'affichage des fichiers en format PDF n'est pas toujours possible avec Explorer (version 5.0). Enfin, sous l'angle de la conception, il faut rappeler qu'il est important de procéder par étape et qu'il est nécessaire de prévoir plus d'une session pour maîtriser tous les outils que, idéalement, l'on voudrait utiliser.

\section{Les avantages}

Au chapitre des avantages, il faut souligner que le professeur a à sa disposition une structure de cours réutilisable et modifiable selon les besoins. La souplesse d'utilisation est ici nettement supérieure à la formule consistant à construire un cours complet sur papier. L'un n'exclut cependant pas l'autre, notre objectif n'est pas de substituer WebCT aux outils traditionnels, mais de les compléter.

L'accès au site à distance constitue également un atout non négligeable. WebCT représente, pour le professeur, un excellent outil de créativité pédagogique, grâce auquel, une fois qu'il le maîtrise, il peut répondre rapidement aux questions formulées par les étudiants.

L'avantage le plus notable, cependant, que nous avons déjà évoqué, reste, dans un cadre d'apprentissage de la traduction, une mise en situation se rapprochant du monde professionnel: usage du courriel et des fichiers joints, respect des délais (l'ordinateur qui tombe en panne est-il encore une bonne excuse?), utilisation raisonnée d'Internet. Le cours de traduction médicale se situant plutôt vers la fin du cursus des étudiants, il devient, comme les autres cours de traduction spécialisée, le lieu privilégié de l'intégration des connaissances antérieures, notamment terminologie, recherche documentaire, utilisation des outils informatiques.

En conclusion, le bilan des avantages et des inconvénients rejoint en grande partie celui qui avait été établi par Lamoureux (1999), mais nous pensons que WebCT permet de répondre, avec une efficacité supérieure aux moyens pédagogiques traditionnels, à des objectifs spécifiques de la matière enseignée.

\section{Développement futur}

Tout d'abord, nous espérons que la réglementation de l'affichage des documents dans un cadre pédagogique à accès restreint nous permettra dans un proche avenir d'enrichir le site de nombreux documents que nous proposons actuellement en format papier à la réserve de la bibliothèque. Ensuite, nous comptons développer la banque de questions dans le cours de langue médicale, et la structurer, si possible, en plusieurs "niveaux", de manière à faciliter l'accès aux connaissances à acquérir en fonction des connaissances préalables. Nous pensons également à étendre l'usage des questionnaires aux cours de traductions, afin de mieux expliciter les types d'erreur couramment rencontrés dans les textes (erreurs de traduction, anglicismes, etc.).

Nous envisageons par ailleurs la possiblité de mettre en place un corpus de textes médicaux sélectionnés, en format électronique, afin de proposer des exercices de recherche informatisée de collocations. Toutefois, pour ce faire, certains obstacles, 
comme l'impossibilité actuelle d'avoir recours à un concordancier dans le site, doivent être levés. Enfin, nous n'excluons pas, notamment dans le cadre des séminaires de maitrise, de refaire une tentative de mise en place de forum de discussion ou de clavardage, mais cela reste, au regard des priorités définies, relativement secondaire.

En conclusion, nous considérons que l'expérience, qui maintenant s'étale sur trois sessions et cinq cours, est très positive. La mise en ouvre des sites nous a paru lourde dans les débuts, mais nous commençons à recueillir le fruit de nos efforts et sommes maintenant convaincue que l'utilisation raisonnée des NTIC dans l'enseignement de la traduction devrait permettre de répondre avec davantage de pertinence aux besoins diversifiés des étudiants.

\section{NOTES}

1. Voir le site américain World Lecture Hall: $<$ http://www.utexas.edu/world/lecture/index.html $>$ et, au Québec, le site francophone de l'Université Laval Rond Point $<$ http://rond-point.ulaval.ca/cours $>$.

2. Université McGill, Université Concordia, Université du Québec à Montréal et Université de Sherbrooke.

3. Voir $<$ http://www.webct.com>.

4. Le programme SUITE (Soutien à l'utilisation d'Internet et des technologies dans l'enseignement), qui relève du Centre d'études et de formation en enseignement supérieur. Voir CÉFES: <http:www. cefes.umontreal.ca $>$.

5. Voir $<$ http://w3.fiu.edu/translation/Courses/SPT4809Web.htm $>$.

6. Les cinq cours de langue et de traduction médicales offerts se répartissent actuellement comme suit: au premier cycle, «Langue médicale et pharmacologique» et «Traduction médicale et pharmacologique»; au deuxième cycle, «Traduction médico-pharmacologique» (DESS), «Traduction biomédicale» et «Traduction pharmaceutique» (en alternance à la maîtrise).

7. Les différentes fonctionnalités offertes par WebCT comprennent notamment: pour l'étudiant, l'accès à un forum de discussion, au courriel électronique, au clavardage, au «tableau blanc» (travail sur un document commun), des exercices d'autoévaluation, un glossaire, la publication des travaux étudiants, les quiz en ligne notés ou non, l'indexation et la recherche dans les contenus, l'annotation des notes de cours, l'affichage des notes, la mise en mémoire de la dernière page consultée, la personnalisation de la page d'accueil, un calendrier; pour le professeur, le suivi individuel des étudiants, l'autocorrection des quiz, la gestion des listes de classe, la gestion des notes, le contrôle de l'accès, la sauvegarde du cours, l'intégration des différents outils, la modification de l'apparence de l'interface (Friesen 2001). Le concepteur choisit celles dont il a besoin.

8. Notre collègue Marie-Claude L'Homme nous a récemment fait connaître un logiciel, portant le nom amusant de HotPotatoes, qui permet de créer des questionnaires de façon similaire à WebCT, mais permettant, en plus, de composer automatiquement des mots-croisés pouvant être affichés dans le site (avec autocorrection). Nous comptons ajouter quelques-uns de ces exercices, qui ajouteront une note ludique à une matière par ailleurs plutôt austère, ce qui, nous l'espérons, devrait faciliter la rétention d'informations.

9. En ce qui nous concerne, nous avons suivi neuf des ateliers offerts par l'Université de Montréal dans le cadre du programme SUITE, soit 21 heures de formation. Les deux auxiliaires qui nous ont aidée à monter le site ont suivi le cours d'introduction (3 heures).

10. Le cours portant sur les outils informatiques faisait déjà appel à un accès par Internet, mais il n'était pas, jusqu'à récemment, intégré à WebCT. D'autres cours de traduction font maintenant appel à WebCT (le cours «Traduction scientifique», par exemple).

11. Subventions obtenues dans le cadre du programme SUITE.

\section{RÉFÉRENCES}

Balliu, C. (1994) : «L'enseignement de la traduction médicale: pour une nouvelle pragmatique», Meta, 39-1, p. 15-25.

Friesen, N. (2001) : Guide to WebCT 3 for Instructors: Design, Development and Delivery, Toronto, McGraw-Hill Ryerson, 152 p. 
Giroux, L. (1999): Le service pédagogique SUITE: Soutien à l'utilisation de l'Internet et des technologies dans l'enseignement - Rapport du comité de travail, Université de Montréal, 52 p. $<$ http://www.suite.umontreal.ca/mission1.html $>$.

Gómez, M. A. et Weinreb, S. (2002): «An Alternative Instructional Model: Teaching Medical Translation Online», Meta, 47-4, p. 643-648.

Lamoureux, J. (1999): Les bénéfices et les problèmes associés à l'utilisation des NYIC dans l'enseignement, Rapport de recherche, Université de Montréal, <http://www.readap.umontreal. $\mathrm{ca} / \mathrm{ntic} \cdot \mathrm{html}>$.

Lee-Jahnke, H. (2001) : «L'enseignement de la traduction médicale : un double défi ? », Meta, 461, p. 145-153.

Rouleau, M. (1994) : La traduction médicale, Brossard, Linguatech.

Vandaele, S. (2001): «Noyaux conceptuels et traduction médicale», Meta, 46-1, p. 16-21.

\section{REMERCIEMENTS}

La conception des sites a été rendue possible grâce à deux subventions du programme SUITE (Université de Montréal) obtenues par l'auteure. Celle-ci remercie les membres de l'équipe du programme pour leur disponibilité et la qualité de la formation sur WebCT. Elle remercie également Micheline Roberge, Deborah Cole et Louise LeBlanc qui ont collaboré de diverses manières à la réalisation du projet, ainsi que les étudiants et étudiantes des cours de langue et de traduction médicales qui ont bien voulu faire part de leurs trouvailles sur la Toile. 